\title{
Antibiogram and Biofilm Formation Among Carbapenem Resistant Klebsiella pneumoniae
}

\author{
Roshani Nhuchhen Pradhan ${ }^{1 *}$, Surendra Kumar Madhup ${ }^{2}$, Shyam Prasad Pant ${ }^{1}$ \\ ${ }^{1}$ Department of Microbiology, St. Xavier's College, Maitighar, Nepal \\ ${ }^{2}$ Dhulikhel Hospital, Dhulikhel, Kavre, Nepal
}

\begin{abstract}
*Corresponding author: Roshani Nhuchhen Pradhan, Department of Microbiology, St. Xavier's College, Maitighar, Nepal. Email: beauty_rosum@yahoo.com
\end{abstract}

\section{ABSTRACT}

Objectives: This cross-sectional study was designed to detect the carbapenemase producing $K$. pneumoniae along with biofilm producers from different clinical specimens and to compare antibiotic susceptibility pattern of biofilm producing carbapenem resistant Klebsiella pneumoniae and biofilm non-producing carbapenem resistant Klebsiella pneumoniae.

Methods: A total of 1475 non-repetitive clinical samples were included on this study. Antibiotic Sensitivity Testing (AST), Modified Hodge Test (MHT) and Modified Carbapenem inactivation method (mCIM) were performed for detection of carbapenemase production and Congo red agar method (CRA) along with Microtitre plate method were performed for detecting biofilm production.

Results: Among the clinical specimens cultured, growth positivity was $62.71 \%$. E. coli was most predominant organism followed by K. pneumoniae (17.89\%). Among the 110 K. pneumoniae, 57 were found to be carbapenemase producer. Majority of the carbapenemase producing K. pneumoniae were isolated from sputum $(45.61 \%)$, in the specimen collected from age group $61-70(28.07 \%)$ and in out-patient department (50.88\%). Similarly, $65.45 \%$ K. pneumoniae out of 110 were found to be biofilm producer by Congo red agar method while among those $72,73.59 \%$ isolates were found to be quantitatively biofilm producer in Microtitre plate assay. Out of 57 carbapenemase producer, $35.08 \%$ were strongly biofilm producer while among 53 carbapenemase non-producer $30.18 \%$ were strongly biofilm producer from Congo red agar method. Moreover, Microtitre plate assay evidenced that, out of 57 carbapenemase producer, $40.35 \%$ were highly biofilm producing and among the 15 carbapenemase nonproducer $66.66 \%$ were highly biofilm producer.

Conclusion: Biofilm formation is highly prevalent with varying degree of resistance among different antibiotics including carbapenems that further augments antibiotic resistance. The study showed carbapenemase producers are stronger biofilm producer than the non-carbapenemase producer. Therefore, it is recommended to identify biofilm formation among carbapenemase producers for effective choice of antibiotics.

Key words: Klebsiella pneumoniae, Carbapenem, Biofilm, Carbapenemase

\section{INTRODUCTION}

Gram negative bacteria are major public health threat. They are becoming resistant to most of the antibiotic drug options available, creating reminiscent situation of the pre-antibiotic era. The most of Gram-negative infections occur in health care setting and are commonly caused by Enterobacteriaceae (mostly Klebsiella pneumoniae), Pseudomonas aeruginosa, and Acinetobacter species. Klebsiella pneumoniae is a Gram-negative bacterium belonging to the family Enterobacteriaceae, the most common cause of community-acquired and hospital-acquired infections. They are responsible in causing several health associated infections which include pneumonia, bloodstream infection, urinary tract infection, wound and surgical site infection, meningitis and so on. The trend of antibiotic resistance is continuously increasing in bacteria that cause either community infections or hospital acquired infections (Shaikh et al. 2014). It leads to increased mortality rate, economic burden, long stay on hospital

Date of Acceptance: November 29, 2019

DOI: https:/ / doi.org/10.3126/tujm.v6i0.26586

Published Online: December, 2019 
and higher medical costs. The higher prevalence of antibiotic resistance and the lack of new antibiotic drug development have constantly reduced the available treatment options for bacterial infections. Emerging resistance in Enterobacteriaceae is a significant problem that requires immediate attention and action. This is a troubling trend, and one that requires vigilance and intensified measures to control the further spread of resistance by these important Gram-negative pathogens (David 2006). Carbapenem resistance is currently rare to most strains of Enterobacteriaceae, but some alarming signs have appeared (David 2006). The spreading of carbapenemase producing Gram negative bacteria in the hospital setting and community is a public health problem with major therapeutic and epidemiological consequences.

Biofilm formation is considered as an important virulence factor of Klebsiella pneumoniae. The majority of Klebsiella pneumoniae infections are associated with biofilm formation on host cells or abiotic surfaces such as indwelling catheter devices. Biofilm producing strains carry high morbidity and mortality in hospitalized patients more specifically patients of Intensive care units (ICU) (Sardi et al. 2013; Deorukhkar et al. 2014). In fact, biofilm formation is another effective way of bacteria for survival in presence of antibiotics (HallStoodley et al. 2004), especially for A. baumannii and Klebsiella pneumoniae which are the most common bacteria for developing biofilm related contamination of medical devices (Singhai et al. 2012). In developing nation like Nepal, where there is rampant irrational use of antibiotics, antibiotics can be easily purchased and used without medical authorization or supervision (Pokharel et al. 2006), the problem of antimicrobial resistance is growing day by day. This study could improve the knowledge regarding the rise and spread of the antimicrobial resistant bacteria in patients. Monitoring the presence of pathogenic and resistant bacteria would be significant in term of providing better and healthy quality of life to the patients with appropriate treatment facilities. Limited studies have been performed for the detection of carbapenem resistant and biofilm formation among the K. pneumoniae from clinical isolates in case of Nepal. This study would be very important step in identifying the prevalence of the carbapenem resistant and biofilm forming $K$. pneumoniae in Nepal. Further, this study would add additional information to existing information. The study was carried out for short duration including limited samples on the particular site only.

\section{MATERIALS AND METHODS}

This study was carried out at Microbiology department of Dhulikhel Hospital, Dhulikhel, Kavre, Nepal. The study was carried out from January to June 2019. A total of 1475 samples were processed out. Ethical approval was obtained from Institutional Review Committee (IRC) of Dhulikhel Hospital. The written and signed inform consents were taken from the patients and the guardian of patients.

Ethics approval and consent to participate: Ethical approval was obtained from Institutional Review Committee (IRC) of Dhulikhel Hospital with the protocol approval number of 103/19.

Sample type and collection methods: Different samples including pus, urine, sputum, catheter tip, tracheal aspirates, and bloods were collected from an outpatient department (OPD) and indoor patient department (IPD). Samples were collected in the sterile container. All the samples were analyzed in microbiology laboratory of the hospital.

Isolation and identification of K. pneumoniae: All the samples were cultured on the MacConkey agar and Blood agar. Growth on the media indicated presences of microorganism. Gram staining was performed for the differentiation of Gram negative bacteria from the Gram positive bacteria. Different biochemical test (indole test, MR-VP test, citrate test, TSIA test and urease test) were performed to identify K. pneumoniae. Confirmed K. pneumoniae isolates were stored for further analysis.

Antibiotic susceptibility testing of $K$. pneumoniae: Antibiotic susceptibility testing was done by applying Kirby Bauer disc diffusion method. During the process, K. pneumoniae isolates were plated on Mueller-Hinton agar and their susceptibilities to carbapenem were checked according to the Clinical and Laboratory Standard Institutes (CLSI) guidelines. Those isolates showing resistant to Meropenem $(10 \mu \mathrm{g})$ were taken for the further confirmatory tests.

Modified carbapenem inactivation method (mCIM) and Modified Hodge test: Modified carbapenem inactivation method (mCIM) was performed following the AST to determine the percentage of isolates that inactivate the carbapenem with production of carbapenemase. To perform the mCIM, a suspension was made by suspending a full $1 \mu$ inoculation loop 
of culture, taken from a Mueller-Hinton or blood agar plate in $2 \mathrm{ml}$ Trypticase soy broth (TSB). Subsequently, a susceptibility-testing disk containing $10 \mu \mathrm{g}$ meropenem was immersed in the suspension and incubated for a minimum of four hours at $35^{\circ} \mathrm{C}$ in ambient air. After incubation, the disk was removed from the suspension using an inoculation loop, placed on a Mueller-Hinton agar plate inoculated with a susceptible $E$. coli indicator strain (ATCC 29522) compared with 0.5 MacFarland tube and subsequently incubated overnight at $35^{\circ} \mathrm{C}$ in ambient air, the zone of inhibition around the meropenem disk was measured and analyzed. Modified Hodge Test (MHT) is a confirmatory test for detection of presence of carbapenemase enzyme in bacteria. During the process, $0.5 \mathrm{McF}$ arland dilution of the E. Coli ATCC 25922 in $5 \mathrm{ml}$ of saline was prepared. Further, it was diluted $1: 10$ by adding $0.5 \mathrm{ml}$ of the 0.5 McFarland to $4.5 \mathrm{ml}$ of saline. A lawn of diluent was streaked on MHA and allowed to dry 3-5 minutes. Meropenem disk $(10 \mu \mathrm{g})$ was placed in the center of the test area. In a straight line, K. pneumoniae was streaked from the edge of the disk to the edge of the plate at 3 different places. Plate was incubated overnight at $35^{\circ} \mathrm{C}$ in ambient air for 16-24 hrs. After 16-24 hours of incubation the plate was checked for a clover leaf-type indentation at the intersection of the K. pneumoniae and the E. coli 25922, within the zone of inhibition of the carbapenem susceptibility disk.

Detection of biofilm production: Biofilm of all the Klebsiella pneumoniae isolates were detected using Congo Red Agar method and Microtitre plate assay. These methods indicated the qualitative and quantitative study of biofilm production respectively. All the isolates were categorized based upon the biofilm formation abilities. In case of Congo red agar, biofilm producer were categorized as strong, moderate, weak and non- producer. The medium composed of Brain heart infusion broth (37 gm/1), sucrose $(5 \mathrm{gm} / 1)$, agar number 1 (10 gm/1) and Congo red dye (0.8 gm/1). Similarly, in case of Microtitre plate assay, the biofilm producer were differentiated as high, moderate, weak and non-adherent based upon the comparison of optical density with positive control. In the study, the highest biofilm producer from the Congo red agar method was taken as the positive control for the Microtitre plate assay. Microtitre plate assay was carried out by inoculating a fresh colony of $K$. pneumoniae in $2 \mathrm{~mL}$ of Brain Heart Infusion broth (BHI). The broth was incubated overnight at $37^{\circ} \mathrm{C}$. Sterile individual plates with 96 flat-bottom polystyrene wells were filled with $200 \mu \mathrm{L}$ of the diluted culture. The plate was incubated at $37^{\circ} \mathrm{C}$ for 48 hours. After incubation, the contents of each well were removed by gentle tapping. The wells were washed with $200 \mu \mathrm{L}$ of phosphate buffer saline $(\mathrm{pH}$ 7.3) to remove free-floating bacteria. Biofilms formed by bacteria adherent to the wells were fixed by $99 \%$ methanol for 5 minutes and stained with $0.1 \%$ crystal violet (CV) with 10-15 minutes. Excess stain was washed gently, and the plate was kept for drying. Around $100 \mu \mathrm{L}$ of $95 \%$ ethanol was added on the well. The optical density of the stained adherent biofilm was measured using a ELISA auto-reader (CHROMATE) at a wavelength of $570 \mathrm{~nm}$.

\section{RESULTS}

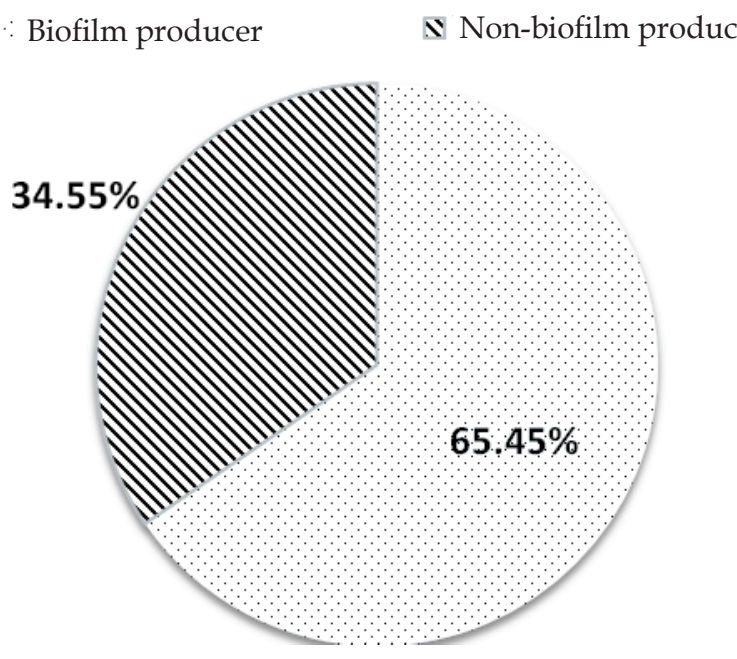

Figure 1: Biofilm producer in Congo red agar 
Table 1: Comparison of biofilm producer and carbapenemase producer

\begin{tabular}{llccc}
\hline & \multicolumn{1}{c}{$\begin{array}{c}\text { Biofilm formation } \\
\text { abilities }\end{array}$} & $\begin{array}{c}\text { Carbapenemase } \\
\text { producer }\end{array}$ & $\begin{array}{c}\text { Carbapenemase non- } \\
\text { producer }\end{array}$ & p-value \\
\hline Congo red agar method & Strong & 20 & 16 & 0.040 \\
& Moderate & 10 & 9 & 8 \\
& Weak & 9 & 20 & \\
& Negative & 18 & 10 \\
Microtitre plate & High & 23 & 2 \\
assay & Moderate & 8 & 2 \\
& Weak & 8 & 1 \\
\hline
\end{tabular}

* $\mathrm{p}$-value was calculated by using the Chi-square test.

Table 2: Antibiotic susceptibility pattern of K. pneumoniae isolates

\begin{tabular}{|c|c|c|c|c|c|c|}
\hline \multirow{2}{*}{ Antibiotics } & \multicolumn{2}{|c|}{ Sensitive } & \multicolumn{2}{|c|}{ Intermediate } & \multicolumn{2}{|c|}{ Resistant } \\
\hline & No. & $\%$ & No. & $\%$ & No. & $\%$ \\
\hline Amikacin & 35 & $44.30 \%$ & 8 & $10.12 \%$ & 36 & $45.56 \%$ \\
\hline Amoxycillin & 36 & $45.56 \%$ & 1 & $1.26 \%$ & 42 & $53.16 \%$ \\
\hline Cefoperazon & 17 & $43.58 \%$ & 0 & 0 & 22 & $56.41 \%$ \\
\hline $\begin{array}{l}\text { Cefoperazone/ } \\
\text { Sulbactam }\end{array}$ & 8 & $22.22 \%$ & 0 & 0 & 28 & $77.77 \%$ \\
\hline Cefixime & 8 & $40.00 \%$ & 2 & $10.00 \%$ & 10 & $50.00 \%$ \\
\hline Ceftriaxone & 26 & $41.93 \%$ & 0 & 0 & 36 & $58.06 \%$ \\
\hline Cotrimoxazole & 33 & $55.93 \%$ & 0 & 0 & 26 & $44.06 \%$ \\
\hline Ciprofloxacin & 15 & $36.58 \%$ & 1 & $2.43 \%$ & 25 & $60.97 \%$ \\
\hline Nitrofurantoin & 2 & $12.50 \%$ & 5 & $31.25 \%$ & 9 & $56.25 \%$ \\
\hline Norfloxacin & 7 & $43.75 \%$ & 0 & 0 & 9 & $56.25 \%$ \\
\hline Piperacillin & 5 & $12.50 \%$ & 4 & $10.00 \%$ & 31 & $77.50 \%$ \\
\hline Gentamicin & 21 & $47.78 \%$ & 1 & $2.27 \%$ & 22 & $50.00 \%$ \\
\hline $\begin{array}{l}\text { Sulphamethoxazole/ } \\
\text { Trimethoprim }\end{array}$ & 7 & $70.00 \%$ & 0 & 0 & 3 & $30.00 \%$ \\
\hline Levofloxacin & 19 & $52.77 \%$ & 0 & 0 & 17 & $47.22 \%$ \\
\hline Cefepime & 16 & $48.48 \%$ & 2 & $6.06 \%$ & 15 & $45.45 \%$ \\
\hline Cefpodoxime & 15 & $45.45 \%$ & 0 & 0 & 18 & $54.54 \%$ \\
\hline Meropenem & 9 & $12.85 \%$ & 0 & 0 & 61 & $87.14 \%$ \\
\hline Imipenem & 9 & $13.23 \%$ & 1 & $1.47 \%$ & 58 & $85.29 \%$ \\
\hline Colistin & 33 & $100 \%$ & 0 & 0 & 0 & 0 \\
\hline
\end{tabular}

During the study period, 1475 non-repetitive samples were processed and $62.71 \%$ showed growth. Out of those, Klebsiella pneumoniae were second most predominant isolates $(17.89 \%)$ after E. coli $(33.98 \%)$. Among the different antibiotics used against $K$. pneumoniae isolates, all the isolates were found to be $100 \%$ sensitive to Colistin. Thus it can be the drug of choice. $87.14 \%$ isolates were resistant to Meropenem and $85.29 \%$ were resistant to Imipenem. Those meropenem resistant K. pneumoniae were phenotypically confirmed to produce carbapenemase by Modified Hodge Test (MHT) and modified Carbapenem Inactivating Method (mCIM). 53.64\% were screened as carbapenemase producer by $\mathrm{mCIM}$ and $51.82 \%$ by MHT. Among the carbapenemase positive Klebsiella pneumoniae isolates, highest percentage producers were observed in sputum $(45.61 \%)$, in age group $61-70(28.07 \%)$ and in outpatient department $(50.88 \%)$. Further, among the total K. pneumoniae, $34.55 \%$ were biofilm producers on CRA. Also, among carbapenemase producer, 35.08\% were strongly biofilm producer and among carbapenemase non-producer $30.18 \%$ were strongly biofilm producer in CRA. In addition to this Microtitre plate assay evidenced that, out of 57 carbapenemase producer, $40.35 \%$ were highly biofilm producing and among the 15 carbapenemase non-producer $66.66 \%$ were highly biofilm producer in Microtitrplate assay. 


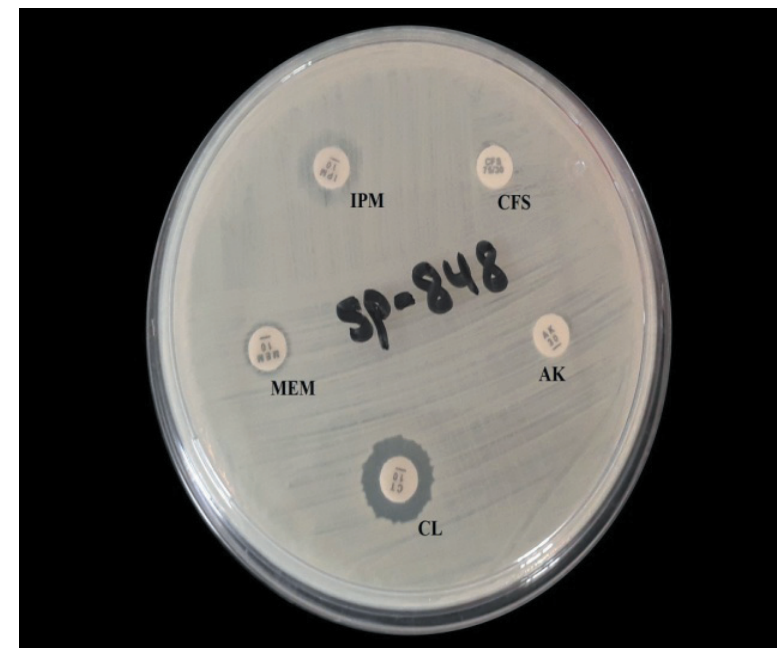

Photograph 1: Antibiotic Susceptibility Test (AST) of Klebsiella pneumoniae showing resistant to Carbapenems

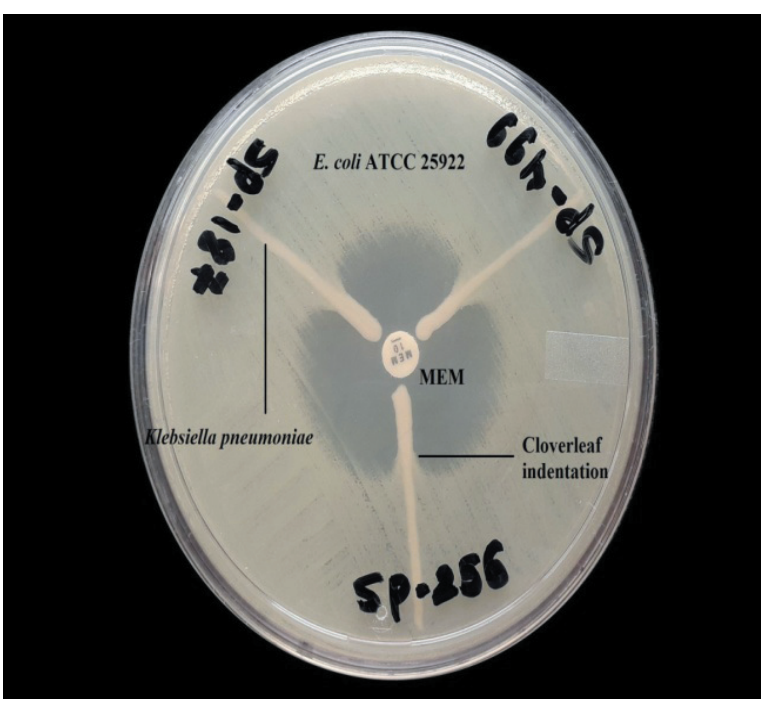

Photograph 3: Modified Hodge Test (MHT) showing the carbapenemase production by the isolates, indicated by cloverleaf indentation (Three different isolates were used for the test in the same plate).

\section{DISCUSSION}

The resistance to carbapenem class of antibiotics which are considered as last resort antibiotics for bacterial infection have cause threat in the health sector. Biofilm is considered as one of the prominent factors for resistance. Hence this study has made attempt to investigate the relationship between carbapenemase producing and biofilm production. Klebsiella pneumoniae was second most predominat after E. coli in our study. However, Bina et al. (2015) and Dahiya et al. (2015) showed Klebsiella pneumoniae to be highly prevalent bacteria in hospital. These distribution and variation

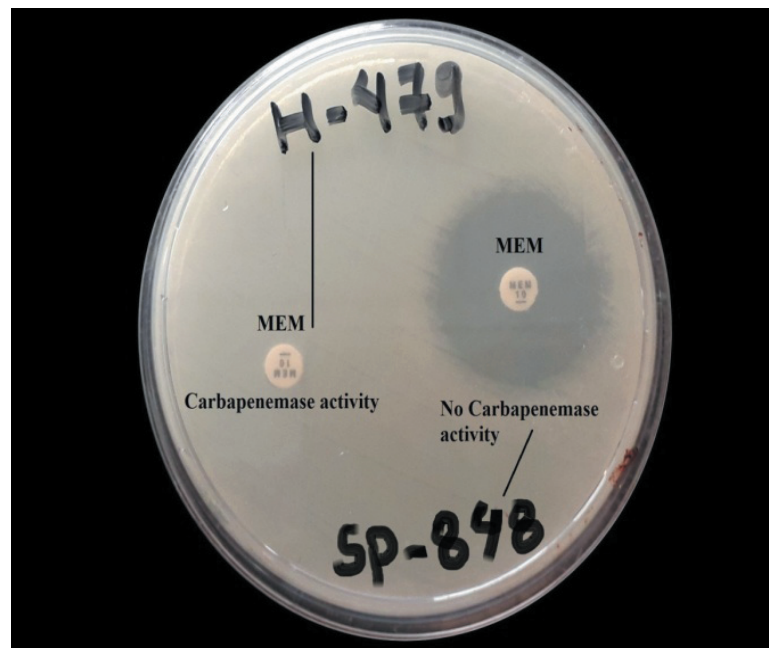

Photograph 2: Modified Carbapenem Inactivation Method (mCIM) showing the carbapenemase activity and no carbapenemase activity (MEM- Meropenem

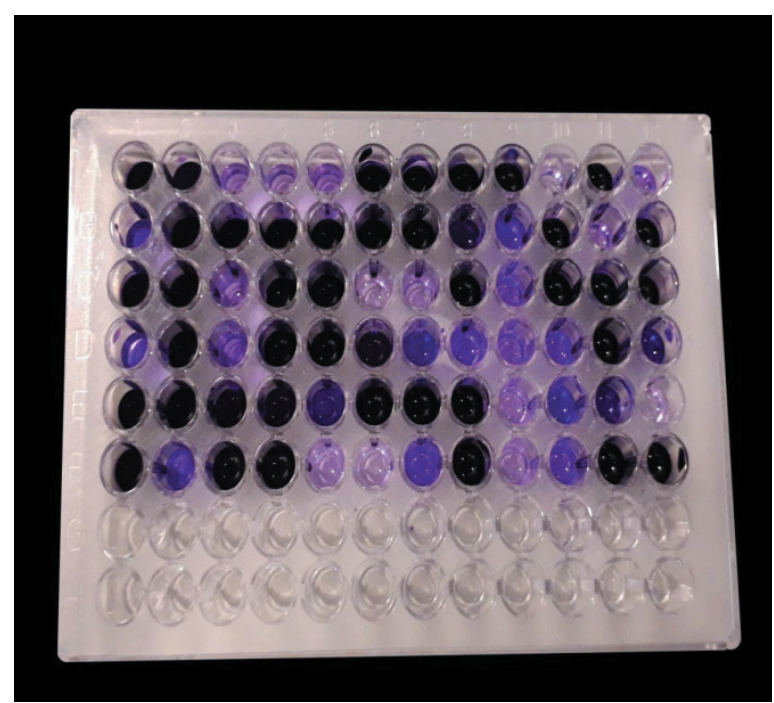

Photograph 4: Detection of biofilm production by Microtitre plate assay (microtitre plate well indicate high, moderate, weak and non-adherent pattern of biofilm formation)

among the bacteria may be due to study location and geographical variation along with adaptation factor in environmental condition that comprises temperature, $\mathrm{pH}$, humidity of the study site. Klebsiella has been associated with different types of infections and one of the important aspects of Klebsiella associated infections is their innate resistance to many antibiotics and the emergence of multidrug resistant strains particularly those involved in nosocomial diseases. AST showed 61 isolates to be meropenem resistant. Using the mCIM, 59 out of 61 isolate showed carbapenemase production with $53.64 \%$ while MHT showed carbapenemase 
production among 57 out of 61 isolates with $51.82 \%$. Those isolates which were negative for MHT and mCIM may have possibility of producing enzymes other than carbapenemase or due to mediation by other mechanism which favor carbapenem resistance or may be due to lesser amounts of carbapenemase production. Among the carbapenemase producer, highest was obtained from sputum. This result is contrast to the study by Henkhoneng et al. (2014) that showed, carbapenem resistant isolates were recovered primarily from urine. The reason for urine as primary samples could be urinary tract infection (UTI), being the most common hospital-acquired infection, which accounts for almost $40 \%$ of all nosocomial infections. But in our study sputum was found to be the key sample for the carbapenemase producer. It may be due to higher number of patient visiting hospital with respiratory tract infection or may be changing trend which substitute respiratory tract infection to be the more common hospital-acquired infection than the UTI. As infants and elderly patients are more prone to immunocompromised conditions, they are more prone to infection by carbapenemase producing isolates and other resistant microorganism that may follow failure antibiotic therapy. Our study also showed higher carbapenemase production in the elderly patients as significant case which might possibly be because of immunosuppressed condition and that may lead to failure of antibiotic application. Further, the oldest group are more likely to be hospitalized than the other ones and the immune status along with the underlying diseases and predisposing factors are more profound in the older age group ( $\geq 60$ years) that makes them more prone to infection. This study showed a significant rate of carbapenem resistance among K. pneumoniae in hospitalized and OPD patients being higher in OPD cases, an indication by shift in infection from hospital acquired infection to community acquired infection. Biofilm-forming bacteria affect millions of people around the world every year, with a high mortality rate. The ability of nosocomial opportunistic microorganisms such as K. pneumoniae to produce biofilms on host-tissue surfaces is a critical stage in the development of infection. Biofilm formation affects the efficacy of antimicrobial therapies and the outcomes of subsequent infections. Thus, early determination of biofilm producer would be helpful to design and use of new and effective approaches which will ultimately aid in the treatment of biofilm-mediated infections and in the reduction of morbidity and mortality in patients suffering from life-threatening nosocomial infections. Our findings indicated that maximum proportion (more than $50 \%$ ) of isolates were biofilm producing strains on Congo red agar (CRA). Among the 72 isolates which were biofilm producers in CRA, 53 isolates only were found to produce biofilm in quantitative assay analysis using Microtiter plate method. Moreover, the interpretation of Microtiter plate assay is optical density dependent and there are chances of subjective errors. However, despite the shortcomings of this method the Microtiter plate assay has been described as the most reliable method for the detection of biofilms (Djordjevic et al. 2002). Most carbapenemase producers are found to be strong biofilm producers. Those isolates which produced more carbapenemase were obtained from the specimen of the patients with higher line of antimicrobial therapy. Those patients have different implants and catheters and which are particularly susceptible to biofilm formation in K. pneumoniae because immune response are highly reduced in proximity to foreign objects (Houri et al. 2016). Proper management of biofilm is essential to control the infection related to carbapenemase production. Management of carbapenemase producing K. pneumoniae infection has become difficult due to limitation of treatment options. However, most of the last line antibiotics including colistin, tigecycline are stable antibiotics even in presence of carbapenemase enzyme. Therefore, this feature allows those antibiotics to be the first choice of the treatment for serious infections with carbapenemase producing K. pneumoniae.

\section{CONCLUSION}

This study identifies the carbapenemase producing Klebsiella pneumoniae and their association with biofilm production. The study revealed that carbapenem resistant isolates are strong biofilm producer than the non-carbapenemase producer. Early detection of biofilm production hence can be useful for clinical decision because of its suggestive

property for potential pathogenic capacity of Klebsiella pneumoniae. Implementation of early detection of carbapenem resistant and biofilm producing isolates would be useful, which may facilitate provision of individualized treatment regimen in patients.

\section{ACKNOWLEDGEMENTS}

We are highly indebted to Department of Microbiology, 
St. Xavier's College, Maitighar and Dhulikhel Hospital for providing us the chance to accomplish the research work. We would like to extend our deepest gratitude to all the participant patients and their guardians without whom this study would never have been completed.

\section{CONFLICT OF INTEREST}

The authors declare no conflict of interest.

\section{REFERENCES}

Djordjevic D, Wiedmann $\mathrm{M}$ and McLandsborough LA (2002). Microtiter plate assay for assessment of Listeria monocytogenes biofilm formation. Appl Environ Microbiol 68(6):2950-2958.

Houri H, Seifi K, Kazemian H, Heidari H, Rezagholizadeh F, Saee $\mathrm{Y}$ and Shirvani F (2016). Evaluation of biofilm formation among Klebsiella pneumoniae isolates and molecular characterization by ERIC-PCR. Jundishapur J Microbiol 9(1): e30682.

Henkhoneng Mate P, et al. (2014). Prevalence of carbapenem resistance among Gram-negative bacteria in a tertiary care hospital in North-East India. IOSR J Dent Med Sci 13(12): 2279-2861.

Dahiya S, Singla P, Chaudhary U and Singh B (2015). Prevalence of Klebsiella pneumoniae Carbapenemase (KPC), metallo beta lactamases and AmpC beta lctamases in clinical isolates of Klebsiella species. Hindawi 4(9): 170-176.

Bina M, Pournajaf A, MirkalantariS, Talebi Mand Irajian G (2015). Detection of the Klebsiella pneumoniae carbapenemase (KPC) in K. pneumoniae isolated from the clinical samples by the phenotypic and genotypic methods. Iran J Pathol 10(3): 199-205.

Singhai M, Malik A, Shahid M, Malik MA and Goyal R. (2012). A study on device-related infections with special reference to biofilm production and antibiotic resistance. J Glob Infect Dis 4: 193-198.

Sardi JCO, Scorzoni L, Bernardi T, Fusco-Almeida AM and Mendes Giannini MJS (2013). Candida species: Current epidemiology, pathogenicity, biofilm formation, natural antifungal products and new therapeutic options. Journal of Medical Microbiology 62(1): 10-24.

Pokhrel BM, Koirala J, Mishra SK, Dahal RK and Tuladhar PKN (2006). Multidrug resistance and extended spectrum $\beta$-lactamase producing strains causing lower respiratory tract and urinary tract infection. J Inst Med 28: 19-27.

Hall-Stoodley L, Costerton JW and Stoodley P (2004). Bacterial biofilms: from the natural environment to infectious diseases. Nat Rev Microbiol 2: 95-108.

Deorukhkar SC and Saini S (2014). Laboratory approach for diagnosis of candidiasis through ages. Int $J$ Curr Microbiol Appl Sci 3(1): 206-218.

David PL (2006). Resistance in gram-negative bacteria: Enterobacteriaceae. Am J Infect Control 34(5): S20-S28.

Shaikh S, Fatima J, Shakil S, Rizvi DMS and Kamal MA (2015). Antibiotic resistance and extended spectrum beta-lactamases: Types, epidemiology and treatment. Saudi J Biol Sci 22(10): 90-101. 